\title{
Mechanisms of Formation of Hemiacetals: Intrinsic Reactivity Analysis
}

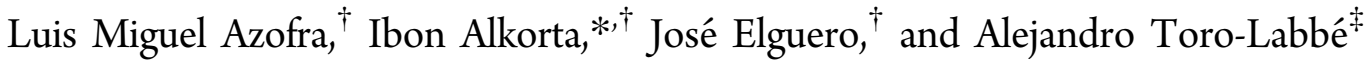 \\ ${ }^{\dagger}$ Instituto de Química Médica, CSIC, Juan de la Cierva 3, E-28006 Madrid, Spain \\ ${ }^{\ddagger}$ Laboratorio de Química Teórica Computacional, QTC, Facultad de Química, Pontificia Universidad Católica de Chile, Casilla 306, \\ E-6094411 Santiago de Chile, Chile
}

Supporting Information

ABSTRACT: The reaction mechanism of the hemiacetal formation from formaldehyde and methanol has been studied theoretically at the B3LYP/6-311+ $+\mathrm{G}(\mathrm{d}, \mathrm{p})$ level. In addition to the study of the reaction between the isolated reactants, three different kinds of catalysis have been explored. The first one examines the use of assistants, especially bridging water molecules, in the proton transfer process. The second one attempts to increase the local electrophilicity of the carbon atom in formaldehyde with the presence of a Brønsted acid $\left(\mathrm{H}^{+}\right.$or $\mathrm{H}_{3} \mathrm{O}^{+}$). The last one considers the combined effect of both catalytic strategies. The reaction force, the electronic chemical potential, and the reaction electronic flux have been characterized for the reaction path in each case. In general, it has been found that structural rearrangements represent an important energetic penalty during the activation process. The barriers for the reactions catalyzed by Brønsted acids show a high percentage of electronic reorganization contribution. The

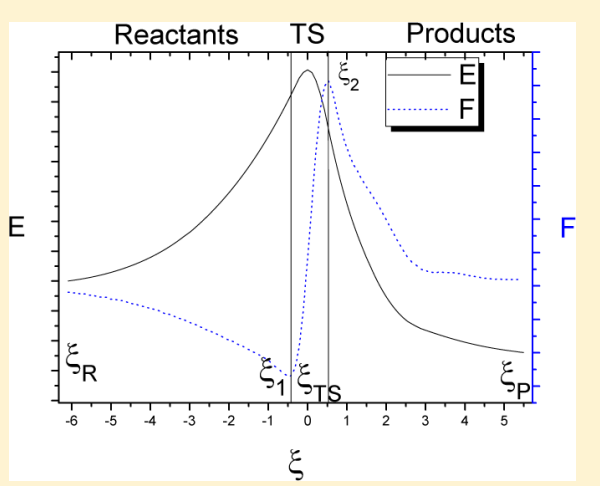
catalytic effects for the reactions assisted by water molecules are due to a reduction of the strain in the transition state structures. The reaction that includes both acid catalysis and proton assistance transfer shows the lowest energy barrier $\left(25.0 \mathrm{~kJ} \mathrm{~mol}^{-1}\right)$.

\section{INTRODUCTION}

From a mechanistic point of view, the reaction of formation of hemiacetals is characterized by two consecutive stages occurring simultaneously: on the one hand, the oxygen atom of a hydroxyl group acts as a nucleophile attacking the carbonyl carbon atom of an aldehyde and forming a covalent $\mathrm{CO}$ bond. On the other hand, the hydrogen atom of the hydroxyl group is transferred to the carbonyl oxygen atom transforming it into a new hydroxyl group. The analogous reaction where the carbonyl compound belongs to a ketone yields a hemiketal. ${ }^{1}$

The addition of nucleophilic agents to carbon atoms of carbonyl groups has been widely studied in the literature. In particular, the catalysis of this process via acid catalysts is an essential part of synthetic organic chemistry. ${ }^{2,3}$ Jencks et al. carried out a detailed study of the mechanism in which a molecule with a hydroxyl moiety attacks another molecule with a carbonyl group in the presence of Brønsted acid HA. Their main conclusion was that the two processes involved, covalent bond $\mathrm{CO}$ formation by nucleophilic attack and proton transfer, are concerted processes, i.e., occurring in one step. ${ }^{3}$ Thus, the key role of the conjugate base $\mathrm{A}^{-}$in the catalytic process was established. Furthermore, Funderburk et al. described the mechanisms of general acid and base catalysis for the reactions of water and alcohols with formaldehyde, ${ }^{4}$ and Meijer and coworkers described theoretically using ab initio molecular dynamics the reaction between formaldehyde and water to give methanediol in the presence of sulfuric acid as catalyst. ${ }^{5}$ In the description of the mechanisms, the presence of a carbocation as intermediate is postulated. The nucleophilic addition of ammonia to formaldehyde in the gas phase and in the complex with formic acid has been studied by Minyaev. ${ }^{6}$ The reactivity of phenolic compounds with formaldehyde has been predicted based on the atomic charges of the isolated phenols. ${ }^{7}$ The intramolecular hemiacetal formation and the importance of the presence of water molecules in the process have been modeled theoretically. ${ }^{8-17}$

The proton transfer process has been widely studied due to its importance in chemical reactivity and biological processes. ${ }^{18-21}$ The acid hydrogen atoms occupy a special position as a promoter and mediator in chemical reactions especially when they occur in solution. $^{22}$ The numerous cases reported can be classified based on the occurring mechanism. For instance, direct proton transfer can be found in the protonated guanine-cytosine base pair of the Watson-Crick chain of DNA, ${ }^{23}$ assistance with hydrogen bonded molecules, like the water, in peptide systems ${ }^{24}$ or carbohydrate systems, in clusters, ${ }^{25}$ occurring in solid and crystalline systems ${ }^{26}$ or even in the excited state. ${ }^{27-31}$

Formaldehyde is the simplest organic compound with a carbonyl group and one of the most important organic compounds in the chemical industry. In addition, formaldehyde is emitted to the troposphere from motor vehicles and industrial emissions. ${ }^{32}$ The properties of this molecule in the ground state

Received: May 9, 2012

Revised: July 9, 2012

Published: July 11, 2012 
(i)

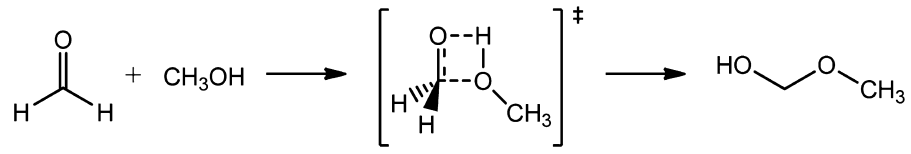

(ii)

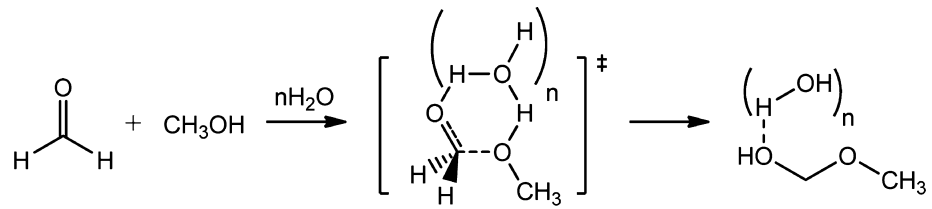

(iii)

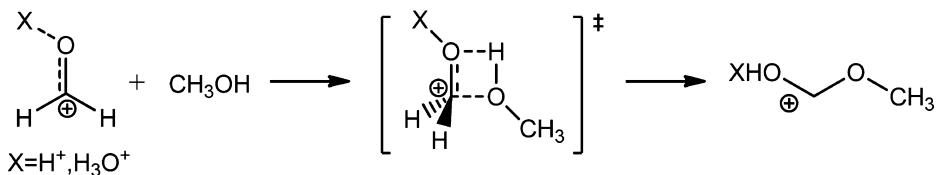

(iv)

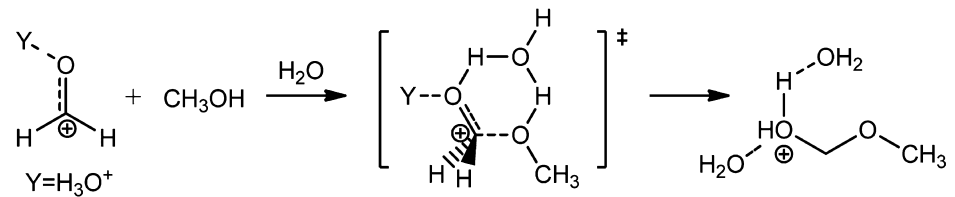

(v)

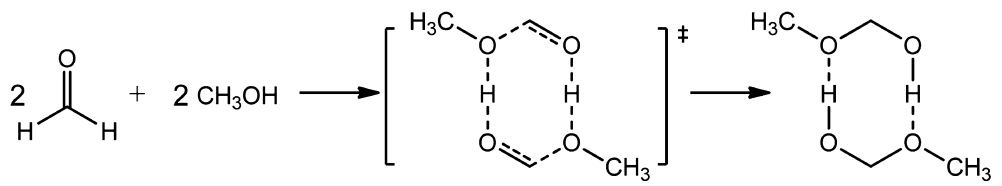

Figure 1. Mechanisms studied for the hemiacetal formation reaction from formaldehyde and methanol: (i) isolated reactants; (ii) assisted with one or two water molecules; (iii) catalyzed by proton or hydronium; (iv) assisted with one water molecule and catalyzed by hydronium; (v) autoassisted.

using ab initio studies were reviewed by Bruna et al..$^{33}$ Besides, Alvarez-Idaboy et al. and Zhao et al. explored the reaction between $\mathrm{H}_{2} \mathrm{CO}$ and the radical $\mathrm{OH}^{34,35}$

In the present article, different mechanisms for the hemiacetal formation from formaldehyde and methanol have been studied (Figure 1). Initially, the reaction between the isolated reactants has been considered (i). The effect on the reaction of the presence of molecules that assist in the proton transfer process (ii and $\mathbf{v}$ ) and the effect of Brønsted acid interacting with the carbonyl group have been explored (iii). Finally, the simultaneous effect of the Brønsted acid and proton assisting molecules have been studied (iv).

\section{THEORETICAL FRAMEWORK}

A chemical reaction can be understood in terms of geometrical changes of the molecular structures and reordering of the electron densities involved in the process. Therefore, identifying structural and electronic changes taking place along the reaction coordinate produces valuable information on the reaction mechanism and can be analyzed by the reaction force concept, ${ }^{36-42}$ which produces a fragmentation of the reaction coordinate and defines regions where specific interactions that might be driving the reaction are turned on and off during the process. The electronic chemical potential, a well-known property for characterizing the reactivity of molecular systems by measuring escaping tendency of electrons from an equilibrium distribution, plays a key role in identifying the electronic activity during a chemical process through its derivative with respect to the reaction coordinate, the so-called reaction electronic flux (REF). ${ }^{43-45}$ Also, the local index of electrophilicity ${ }^{46}$ has been used to describe the behavior of this property in the carbon atom of formaldehyde in cationic systems.

Energy and Reaction Force. A transition state is a chemical entity along the intrinsic reaction coordinate $(\text { IRC }=\xi)^{47,48}$ which is a stationary point and presents one imaginary frequency. It connects reactants and products through the path of minimum potential energy in a one-step chemical reaction. The energy profile does not give us complete information about the reaction mechanism and therefore, it is interesting to consider the force of the reaction, $F(\xi)$, defined as the negative derivative of the total energy, $E$, with respect to the reaction coordinate

$$
F(\xi)=-\frac{\mathrm{d} E}{\mathrm{~d} \xi}
$$

According to the Transition State Theory ${ }^{49}$ (TST), the energy profile of an elementary step presents three critical points: two minima, one for the reactants $\left(\xi_{\mathrm{R}}\right)$ and another for the products $\left(\xi_{\mathrm{p}}\right)$, and one maximum for the transition state $\left(\xi_{\mathrm{TS}}\right)$ (Figure 2$)$. In addition, $F(\xi)$ exhibits two very important critical points: a minimum at $\xi_{1}$ and a maximum at $\xi_{2}$. Thus, we can define three regions: the first one associated with the reactants $\left[\xi_{\mathrm{R}}, \xi_{1}\right]$ in which the reactants are prepared for the reaction mainly through structural reordering. The second one, limited by $\xi_{1}$ and $\xi_{2}$, where the TS is located, corresponds to the region where most formation and breaking of the bonds take place. This region is mainly associated to an electronic reordering. Finally, the third region, between $\xi_{2}$ and $\xi_{\mathrm{P}}$, is associated with structural relaxation to reach the products of the reaction. ${ }^{36-42}$ Note that $\xi_{1}<\xi_{\mathrm{TS}}<\xi_{2}$.

The reaction force analysis provides an energy partition of the activation barrier. Thus, $W_{1}$ represents the amount of energy required to reorganize the system geometrically, while $W_{2}$ 


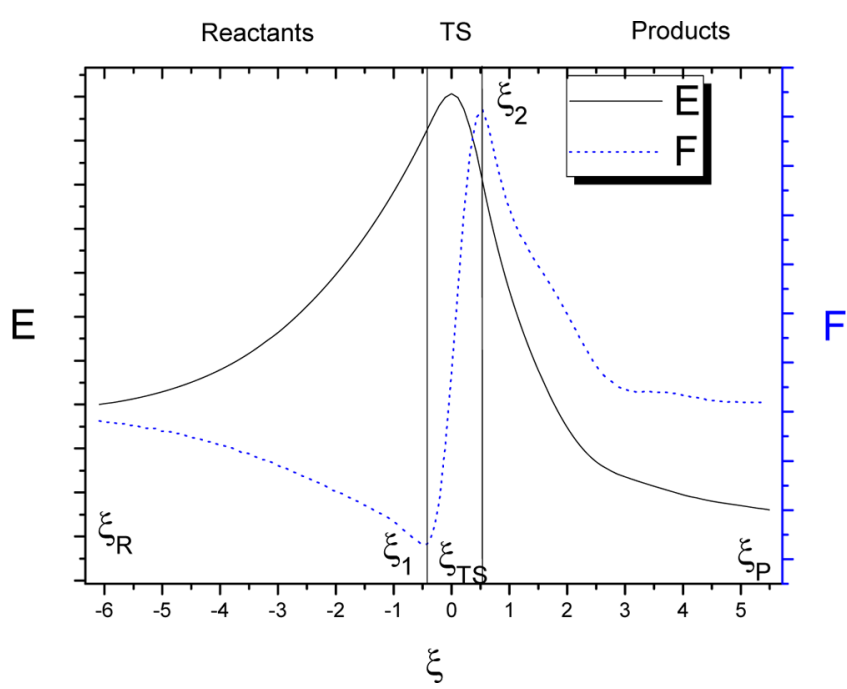

Figure 2. Solid and dotted lines represent a generic energy profile and force of the reaction, $F(\xi)$, vs the reaction coordinate. The location of the stationary points of the energy and $F(\xi)$ are indicated. Two vertical lines separate the reactants region (left), the transition state region (center), and the products region (right).

represents the electronic changes in the reactant to reach the transition state form.

$$
\begin{aligned}
& \Delta E^{*}=W_{1}+W_{2} \\
& W_{1}=-\int_{\xi_{0}}^{\xi_{1}} F(\xi) \mathrm{d} \xi>0 \text { and } W_{2}=-\int_{\xi_{1}}^{\xi_{\mathrm{TS}}} F(\xi) \mathrm{d} \xi>0
\end{aligned}
$$

Chemical Properties Based on Conceptual DFT. Conceptual DFT ${ }^{50,51}$ offers a range of theoretical tools that allow to study and understand changes at the electronic level directly associated with physicochemical properties of the entities that react chemically. In this case, the electronic chemical potential, $\mu$, the electronegativity, $\chi$, chemical hardness, $\eta$, the electrophilic power of the molecule, $\omega$, Fukui functions, and reaction electronic flux, $J(\xi)$, will be mentioned.

The electronic chemical potential, $\mu$, for a system of $N$ electrons is defined as the derivative of the total energy with respect to $N$ when the external potential, $v(\vec{r})$, remains constant (eq 4). Considering that the number of electrons, $N$, is a discontinuous variable, the electronic chemical potential can be approximated through the application of finite differences and Koopmans' theorem ${ }^{52}$ as the negative sum of the first ionization potential, $I$, and the electron affinity, $A$, divided by two. In addition, these two parameters, $A$ and $I$, can be approximated by the values of the highest occupied and lowest unoccupied molecular orbitals, $\varepsilon_{\mathrm{H}}$ and $\varepsilon_{\mathrm{L}}$, respectively. The electronegativity, ${ }^{53} \chi$, is the opposite value of $\mu$.

$$
\mu=-\chi=\left(\frac{\partial E}{\partial N}\right)_{v(\vec{r})} \approx-\left(\frac{I+A}{2}\right) \approx \frac{\varepsilon_{\mathrm{L}}+\varepsilon_{\mathrm{H}}}{2}
$$

The chemical hardness, ${ }^{54,55} \eta$, is defined as the second derivative of the total energy with respect to $N$ when $v(r)$ is constant (eq 5). Again, by applying the finite differences approximation and Koopmans' theorem, it can be approximated by the difference between the energy of the frontier orbitals. The chemical softness, $S$, is the inverse of hardness.

$$
\eta=S^{-1}=\left(\frac{\partial^{2} E}{\partial N^{2}}\right)_{v(\vec{r})} \approx-(I-A) \approx \varepsilon_{\mathrm{L}}-\varepsilon_{\mathrm{H}}
$$

The electrophilic power, $\omega$, was introduced by Parr et al., ${ }^{46}$ and its formulation is analogous to that of the power in classical electricity (eq 6):

$$
\omega \approx \frac{\mu^{2}}{2 \eta} \approx \frac{(I+A)^{2}}{8(I-A)} \approx \frac{\left(\varepsilon_{\mathrm{L}}+\varepsilon_{\mathrm{H}}\right)^{2}}{8\left(\varepsilon_{\mathrm{L}}-\varepsilon_{\mathrm{H}}\right)}
$$

The global properties defined, so far, are useful in monitoring intrinsic changes in the molecules. However, the reactivity of the molecules resides on the atomic centers and consequently local properties are desirable. They can be calculated from the Fukui function, ${ }^{56} f(\vec{r})$, which is defined as the second derivative of the total energy with respect to $N$ and $v(\vec{r})$ (eq 7). This function complies an important property: its integration over all space gives the unit value.

$$
f(\vec{r})=\left[\frac{\partial^{2} E}{\partial N \delta v(\vec{r})}\right] \rightarrow \int_{\Omega} f(\vec{r}) \mathrm{d} r=1
$$

Because of the discrete nature of $N$, two Fukui functions are derived, the nucleophilic, $f^{+}(\vec{r})$, and the electrophilic, $f^{-}(\vec{r})$, functions. They are approximated by the electron density of the LUMO and HOMO frontier orbitals, respectively. Both, nucleophilic and electrophilic Fukui functions can be evaluated for each atom as indicated in eqs 8 and 9, respectively.

$$
\begin{aligned}
& f^{+}(\vec{r})=\left|\phi^{\mathrm{L}}(\vec{r})\right|^{2}=\rho^{\mathrm{L}}(\vec{r}) \rightarrow f_{k}^{+}=p_{k}(N+1)-p_{k}(N) \\
& f^{-}(\vec{r})=\left|\phi^{\mathrm{H}}(\vec{r})\right|^{2}=\rho^{\mathrm{H}}(\vec{r}) \rightarrow f_{k}^{-}=p_{k}(N)-p_{k}(N-1)
\end{aligned}
$$

where the subscript $k$ refers to a particular atom $k$. Then, $p_{k}(N)$ is the electronic population on an atom $k$ in the neutral molecule, $p_{k}(N+1)$ is the electronic population on an atom $k$ in the radical anion molecule, and $p_{k}(N-1)$ is the electronic population on an atom $k$ in the radical cation molecule.

Using the nucleophilic Fukui function and the global electrophilicity, the local electrophilicity index, $\omega_{k}$, is obtained (eq 10):

$$
\omega_{k}=f_{k}^{+} \omega
$$

The maximum electrophilicity power in a molecule will be present at the site where the Fukui function for a nucleophilic attack $f_{k}^{+}$displays its maximum value, i.e., at the active site of the electrophile. $^{57}$

Finally, the reaction electronic flux, $J(\xi)$, associated with a chemical reaction can be defined using eq 11 :

$$
J(\xi)=-\frac{\mathrm{d} \mu}{\mathrm{d} \xi}
$$

The interpretation of the reaction electronic flux results from the analogy with classical thermodynamics. Positive values of $J(\xi)$ should be associated to spontaneous rearrangements of the electron density driven by bond strengthening or forming processes; negative values of $J(\xi)$ are indicating nonspontaneous rearrangements of the electron density that are mainly driven by bond weakening or breaking processes. ${ }^{58}$ 


\section{COMPUTATIONAL DETAILS}

All the geometries employed in this study have been fully optimized with the hybrid B3LYP ${ }^{59,60}$ density functional and Pople's basis set $6-311++\mathrm{G}(\mathrm{d}, \mathrm{p}) .{ }^{61}$ The Synchronous TransitGuided Quasi-Newton (STQN) Methods ${ }^{62}$ (QST2 and QST3) have been used to locate the transition states. The intrinsic reaction coordinate (IRC) procedure in which the reaction coordinate, $\xi$, is expressed in mass-weighted internal coordinates ${ }^{63}$ was used. Frequency calculations have been carried out in all the stationary geometries in order to verify that they correspond to an energetic minimum or transition state. All calculations were performed with the Gaussian09 package. ${ }^{64}$ The Natural Bond Orbital (NBO) methodology ${ }^{65}$ has been employed to calculate the electronic population on the atomic centers with the NBO 3.1 program. ${ }^{66}$ The electron density of the systems has been analyzed by means of Atoms In Molecules (AIM) methodology ${ }^{67,68}$ with the Morphy program. ${ }^{69,70}$

\section{RESULTS AND DISCUSSION}

In the present paper, the reaction mechanism of the hemiacetal formation between isolated formaldehyde and methanol will be discussed. In addition, the assistance of the proton transfer by the presence of one or two bridging water molecules, the effect of the presence of Brønsted acids $\left(\mathrm{H}^{+}\right.$or $\left.\mathrm{H}_{3} \mathrm{O}^{+}\right)$in order to increase the local electrophilicity of the carbon atom of formaldehyde, and the combination of the effects of both catalytic strategies have been examined. Figure 1 illustrates the five mechanisms studied.

The first two subsections will discuss some global parameters of the reaction: energy and reaction force profiles and chemical potential and reaction electronic flux. The last subsection will consider the local index of electrophilicity of the carbon atom of the carbonyl group since it is the only heavy atom that acts as an electrophile in all the reactions considered here.

Energy and Reaction Force Profiles. The total activation energy, geometric and electronic partitions, and the reaction energy have been gathered in Table 1 . Table 2 includes the values

Table 1. Activation Energy, Geometric Partition Activation Energy, Electronic Partition Activation Energy and Reaction Energy in $\mathrm{kJ} \mathrm{mol}^{-1}$ for the Different Mechanisms Calculated at the B3LYP/6-311++G(d,p) Level ${ }^{a}$

\begin{tabular}{lllll}
\multicolumn{1}{c}{ type } & $\Delta E^{\ddagger}$ & \multicolumn{1}{c}{$W_{1}$} & \multicolumn{1}{c}{$W_{2}$} & $\Delta E_{\mathrm{R}}$ \\
i & 141.3 & $124.9(88)$ & $16.4(12)$ & -47.9 \\
ii $\left(\mathrm{H}_{2} \mathrm{O}\right)$ & 74.7 & $65.8(88)$ & $8.9(12)$ & -47.8 \\
ii $\left(2 \mathrm{H}_{2} \mathrm{O}\right)$ & 78.4 & $73.2(93)$ & $5.3(7)$ & -34.1 \\
iii $\left(\mathrm{H}^{+}\right)$ & 99.9 & $64.1(64)$ & $35.8(36)$ & 11.9 \\
iii $\left(\mathrm{H}_{3} \mathrm{O}^{+}\right)$ & 86.5 & $62.3(72)$ & $24.2(28)$ & -5.6 \\
iv & 25.0 & $16.2(65)$ & $8.8(35)$ & -9.7 \\
v & 61.2 & $42.6(69)$ & $18.7(31)$ & -75.3
\end{tabular}

${ }^{a}$ In parentheses, percentage with respect to the activation energy.

of the critical points of the energy and reaction force. The figures of the energy and reaction force profiles of all the studied reactions are reported in the Supporting Information.

The activation energy for the reaction of isolated formaldehyde and methanol is very high $\left(141.3 \mathrm{~kJ} \mathrm{~mol}^{-1}\right)$. Of this amount, $88 \%$ is due to the geometric rearrangement, and the remaining $12 \%$ corresponds to the electronic reordering. The reaction force value at $\xi_{1}$ is $-54.0 \mathrm{~kJ} \mathrm{~mol}^{-1} \mathrm{amu}^{-1 / 2} \mathrm{bohr}^{-1}$. This high value is due to instability of the activated reactant since a strained four-membered ring is generated. In the reactions
Table 2. Intrinsic Reaction Critical Points of the Energy $\left(\xi_{\mathrm{R}}\right.$ and $\left.\xi_{\mathrm{p}}\right)$ and of the Reaction Force $\left(\xi_{1}\right.$ and $\left.\xi_{2}\right)$ in amu $u^{1 / 2}$ bohr; by Definition, $\xi_{\mathrm{TS}}=0.00 \mathrm{amu}^{1 / 2} \mathrm{bohr}$

\begin{tabular}{lcccc}
\multicolumn{1}{c}{ type } & $\xi_{\mathrm{R}}$ & $\xi_{1}$ & $\xi_{2}$ & $\xi_{\mathrm{P}}$ \\
i & -6.09 & -0.43 & 0.53 & 5.50 \\
ii $\left(\mathrm{H}_{2} \mathrm{O}\right)$ & -4.06 & -0.42 & 0.43 & 4.25 \\
ii $\left(2 \mathrm{H}_{2} \mathrm{O}\right)$ & -7.46 & -0.31 & 0.53 & 5.94 \\
iii $\left(\mathrm{H}^{+}\right)$ & -3.18 & -0.62 & 0.41 & 4.03 \\
iii $\left(\mathrm{H}_{3} \mathrm{O}^{+}\right)$ & -5.15 & -0.52 & 0.41 & 7.67 \\
iv & -4.86 & -1.19 & 1.06 & 10.91 \\
$\mathbf{v}$ & -9.28 & -1.94 & 0.63 & 7.10 \\
\hline
\end{tabular}

assisted by one or two water molecules, it is found that the barriers decrease to $65.8 \mathrm{~kJ} \mathrm{~mol}^{-1}$ and $73.2 \mathrm{~kJ} \mathrm{~mol}^{-1}$, respectively. The reaction force values at $\xi_{1}$ with one and two water molecules are $-27.4 \mathrm{~kJ} \mathrm{~mol}^{-1} \mathrm{amu}^{-1 / 2} \mathrm{bohr}^{-1}$ and $-23.9 \mathrm{~kJ} \mathrm{~mol}^{-1} \mathrm{amu}^{-1 / 2}$ bohr $^{-1}$, respectively. These values indicate the catalytic effect of the increment of the size of the ring generated in the activated reactant, either six-membered ring with one bridging water molecule or eight-membered ring with two bridging water molecules, minimize the angular strain. ${ }^{71}$ In any case, the percentage of the geometric activation term remains similar to that found in the reaction mechanism between the isolated reactants, $\mathbf{i}$.

The barriers of the reaction, where the electrophilicity power of the carbon atom in formaldehyde is enhanced by the presence of the Brønsted acids $\mathrm{H}^{+}$and $\mathrm{H}_{3} \mathrm{O}^{+}$, are $99.9 \mathrm{~kJ} \mathrm{~mol}^{-1}$ and $86.5 \mathrm{~kJ}$ $\mathrm{mol}^{-1}$, respectively. As expected, the Brønsted acids act as catalysts of the reaction. The reaction force values at $\xi_{1}$ are the largest calculated in absolute term, with values of $-81.4 \mathrm{~kJ} \mathrm{~mol}^{-1}$ $\mathrm{amu}^{-1 / 2} \mathrm{bohr}^{-1}$ and $-67.5 \mathrm{~kJ} \mathrm{~mol}^{-1} \mathrm{amu}^{-1 / 2} \mathrm{bohr}^{-1}$, respectively. In these activated reactants, a strained four-membered ring is formed, and in addition, the previous protonation of the oxygen atom in the carbonyl group converts it in a poor base, which decreases the ability of the carbonyl oxygen to accept the hydrogen atom. Once the system is in the transition state region, the electronic rearrangement takes a very important weight with a value of $36 \%$ for iii $\left(\mathrm{H}^{+}\right)$and a $28 \%$ for iii $\left(\mathrm{H}_{3} \mathrm{O}^{+}\right)$of the total amount of the activation energy.

Mechanism iv can be defined as the integration of mechanism ii $\left(\mathrm{H}_{2} \mathrm{O}\right)$ and mechanism iii $\left(\mathrm{H}_{3} \mathrm{O}^{+}\right)$and corresponds approximately to the sum of the principal catalytic characteristics of both. On the one hand, the presence of a water molecule causes the reaction force value at $\xi_{1}$ to be $-16.5 \mathrm{~kJ} \mathrm{~mol}^{-1} \mathrm{amu}^{-1 / 2}$ bohr $^{-1}$, which is the second smallest value in absolute value of this property obtained in the present work. On the other hand, once in the transition state area, the percentage of electronic reorganization energy is $35 \%$ as in the case of the iii profile.

In mechanism $\mathbf{v}$, where two pairs of reactants interact forming a $C_{i}$ symmetry tetramer and the proton transfer is assisted by the second pair of reactants, we found the smallest value of the barrier in the neutral reaction considered here $\left(61.2 \mathrm{~kJ} \mathrm{~mol}^{-1}\right)$, and in contrast with the results observed in the rest of neutral mechanisms, $69 \%$ of this value is associated to the geometric rearrangement, and the remaining $31 \%$ corresponds to the electronic reordering.

All these results allow us to reach three conclusions: (a) systems that have a TS structure formed by strained cycles present a high value on the reaction force during the geometric rearrangement; (b) the geometric rearrangement supposes a greater energy penalty than the electronic reordering; (c) mechanism iv shows the best values from a catalytic point of view 

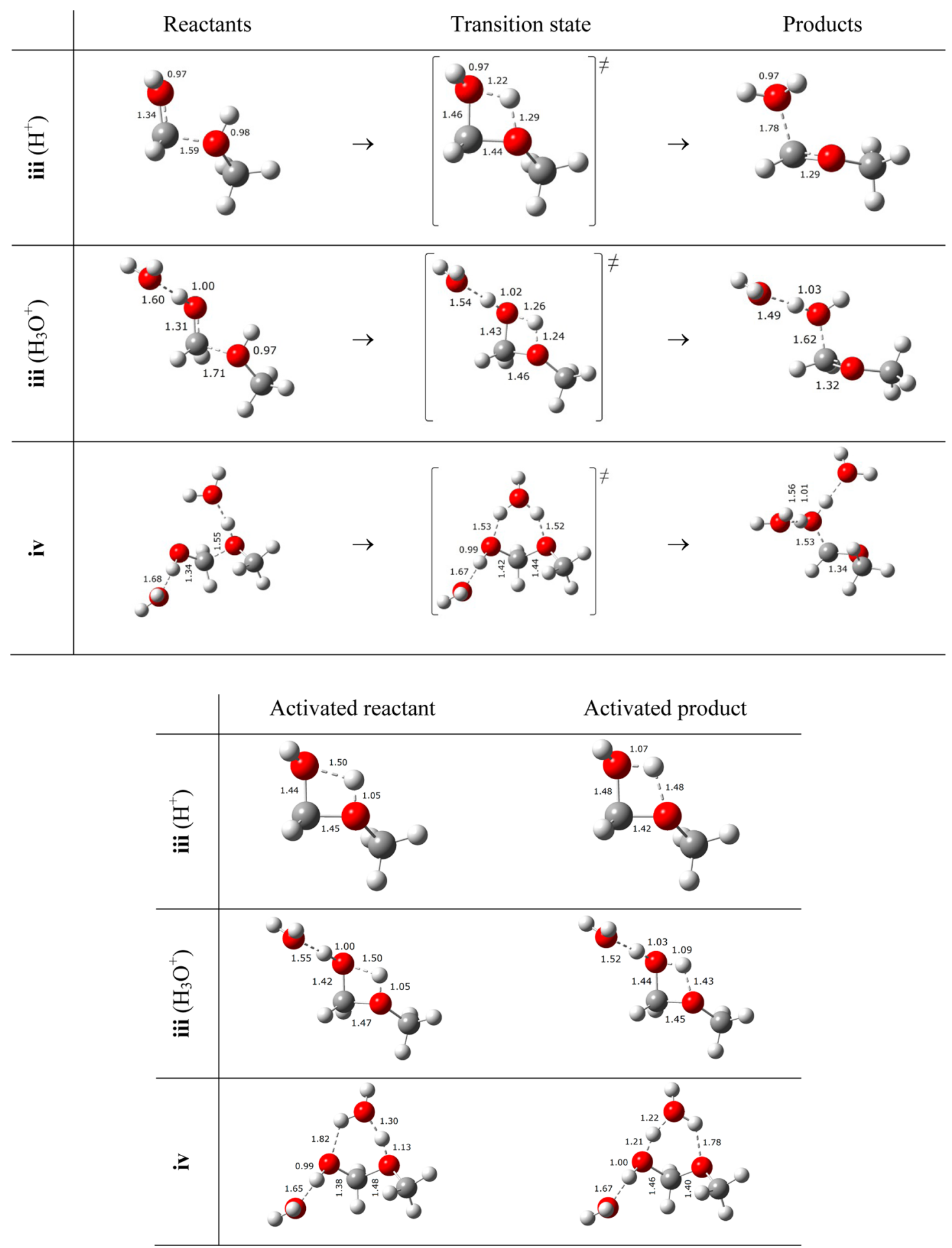

Figure 3. Geometries of the stationary points of the reactions (energy and reaction force) catalyzed by Brønsted acids (selected distances in $\AA$ ) calculated at the B3LYP/6-311++G(d,p) computational level.

since it incorporates the advantages of the two individual catalytic mechanisms, bridging water molecule and Brønsted acid.

The most significant geometrical changes along the reaction path in the mechanism catalyzed by Brønsted acids are shown in Figure 3. In the first place, it is observed that the double bond character of the carbonyl group in the reactant is lost in the presence of a positive charge Brønsted acids, presenting the carbon atom a $s p^{3}$ hybridization.
The TS geometries show an increasing values of the $\mathrm{O} \cdots \mathrm{H}$ distance along the series iii $\left(\mathrm{H}^{+}\right)<\mathrm{iii}\left(\mathrm{H}_{3} \mathrm{O}^{+}\right)<\mathrm{iv}$ and very short $\mathrm{C} \cdots \mathrm{O}$ distances especially when compared to those obtained in the TS of the neutral reactions that range between 1.79 and 1.67 A. A high similarity between the structures of the stationary points of the reaction force (at $\xi_{1}$ and $\xi_{2}$ ) and the structure of the TS is observed. The most significant changes are located in the shortening of the $\mathrm{CO}$ bond being formed, in the increasing of the $\mathrm{C}=\mathrm{O}$ distances, and in the migration of the hydrogen atoms. 

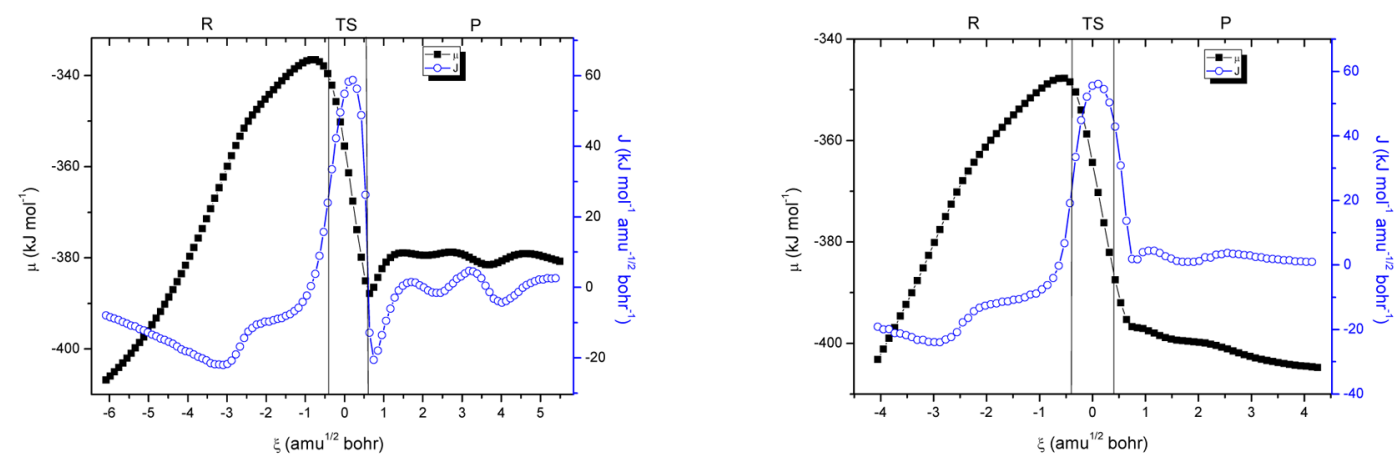

i
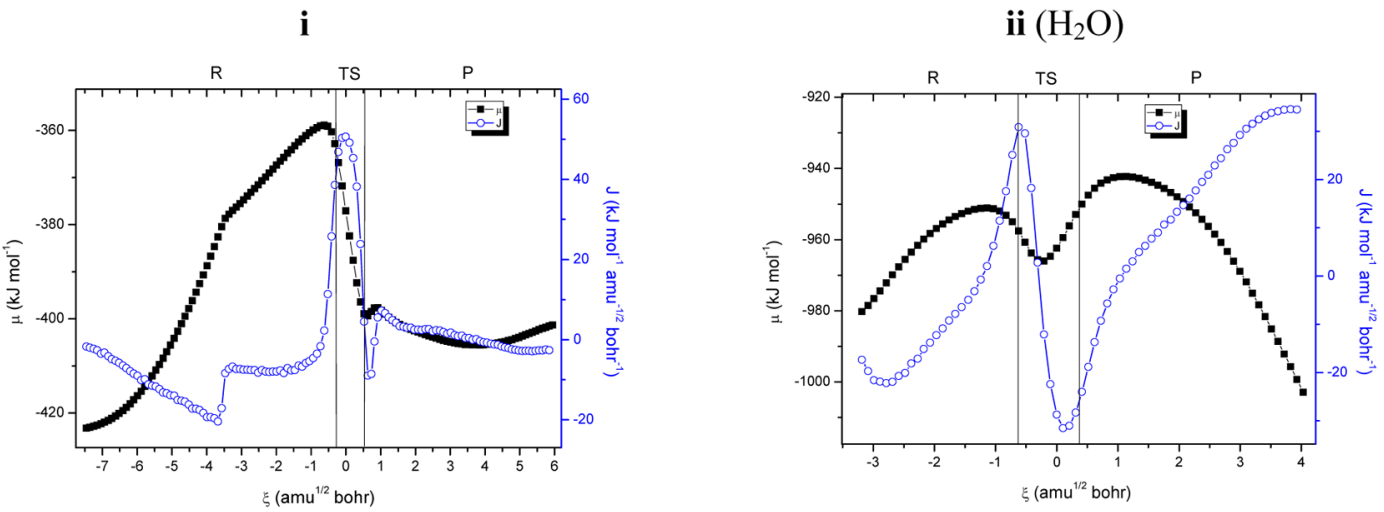

iii $\left(\mathrm{H}^{+}\right)$

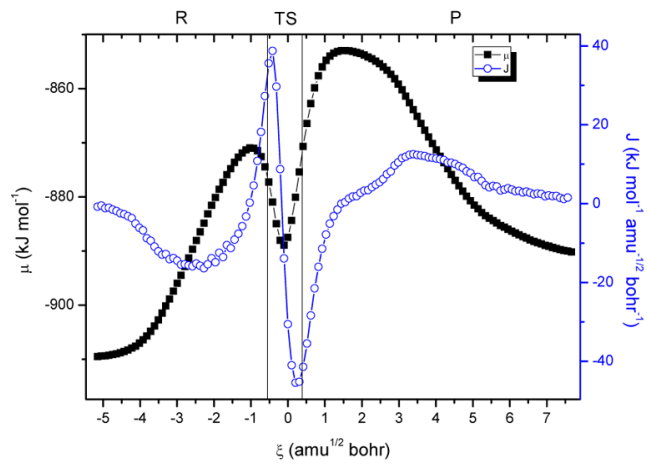

iii $\left(\mathrm{H}_{3} \mathrm{O}^{+}\right)$

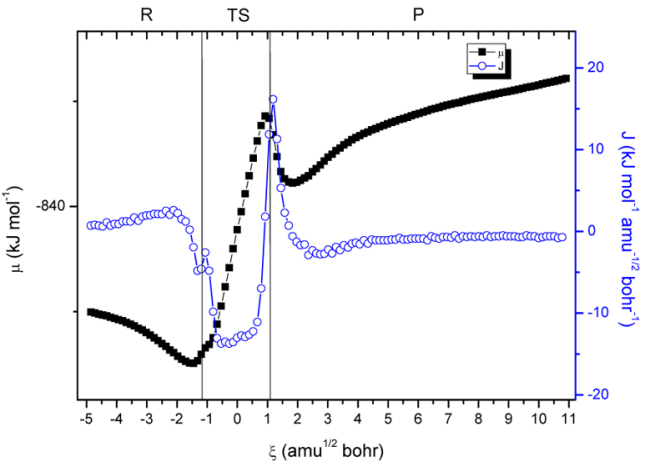

iv

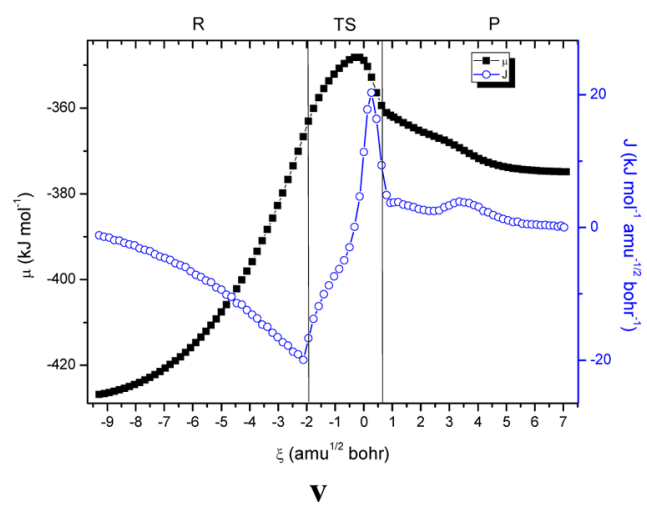

Figure 4. In black and with squares, the electronic chemical potential profile, and in blue and with circles, the reaction electronic flux (REF) profile for the different mechanism studied. Two vertical lines separate the reactants region (left), the transition state region (center), and the products region (right).

The geometries of the stationary points (energy and reaction force) of the reactions $\mathbf{i}$, ii (both $\mathrm{H}_{2} \mathrm{O}$ and $2 \mathrm{H}_{2} \mathrm{O}$ ), and $\mathbf{v}$ and the geometries (coordinates) of all the TS are reported in the Supporting Information.
In the products, it is observed a dissociation of the water molecule formed from the carbonyl oxygen moiety. This effect is much more pronounced in the case of $\mathrm{iii}\left(\mathrm{H}^{+}\right)$where the distance between the carbon atom of the product and the oxygen of the 

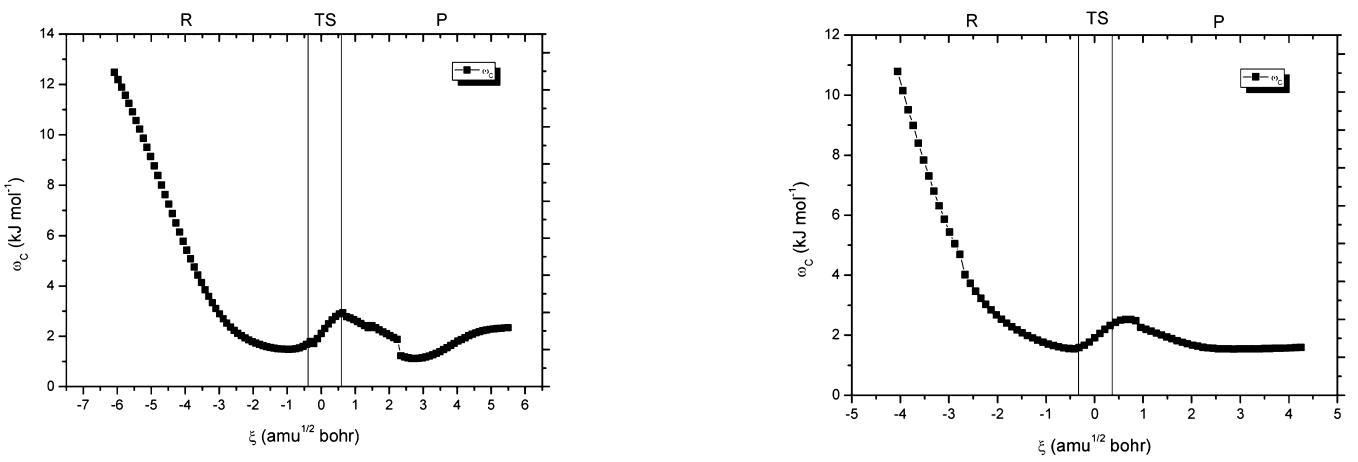

i
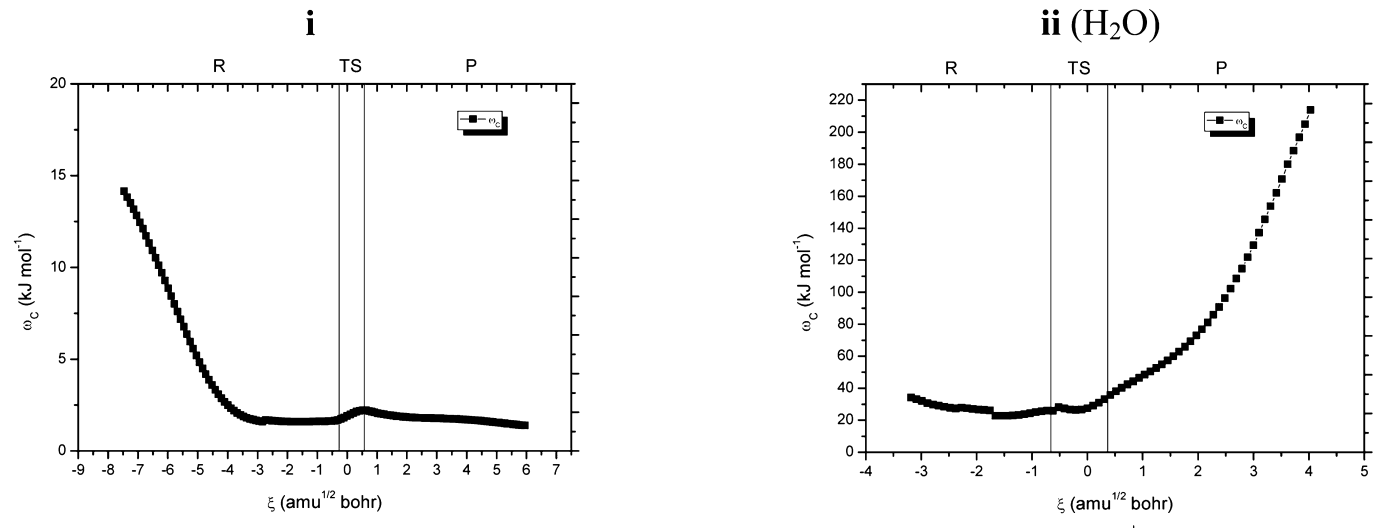

ii $\left(2 \mathrm{H}_{2} \mathrm{O}\right)$

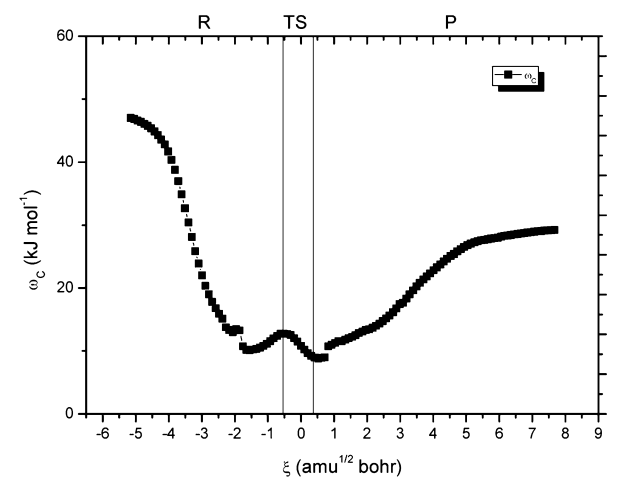

iii $\left(\mathrm{H}^{+}\right)$

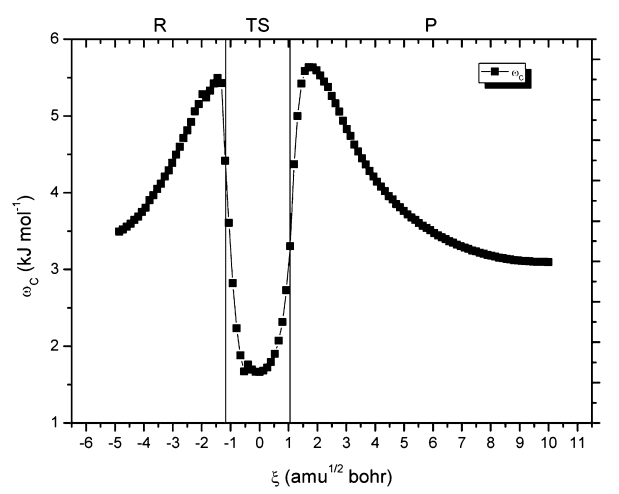

iv

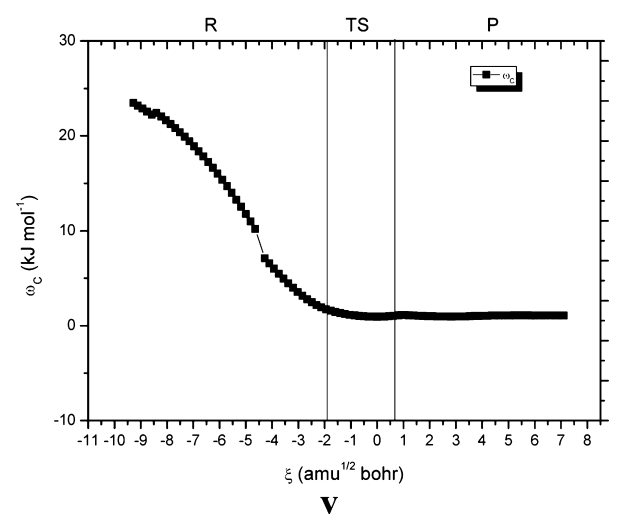

Figure 5. Local electrophilicity profile of a carbon atom in formaldehyde for the different reaction mechanisms. Two vertical lines separate the reactants region (left), the transition state region (center), and the products region (right).

leaving $\mathrm{H}_{2} \mathrm{O}$ molecule is $1.78 \AA$. The intermolecular distance decreases with the presence of water molecules, thus, it amounts to $1.62 \AA$ when one water molecule is included [reaction iii $\left.\left(\mathrm{H}_{3} \mathrm{O}^{+}\right)\right]$and to $1.56 \AA$ in reaction iv. The values of the electron density and Laplacian at the $\mathrm{CO}$ bond critical point indicate an evolution from weak interaction to covalent bond in the $\mathrm{C} \cdots \mathrm{OH}_{2}$ contact from the first to the third cases mentioned before: 0.103 , 0.152 , and 0.141 au for the electron density and $0.108,-0.060$, 
and -0.237 au for the Laplacian in each case. A similar feature in the protonation of alcohols has been described theoretically and used experimentally as an effective way to generate carbocations in the gas phase. ${ }^{72,73}$

Chemical Potential and Reaction Electronic Flux. The evolution of the electronic chemical potential along the intrinsic reaction coordinate provides important information in terms of the electronic activity of the reaction (Figure 4). On the basis of the region where the most noticeable changes can take place, the reactions studied can be classified in four types: (a) reactions $\mathbf{i}$, ii $\left(\mathrm{H}_{2} \mathrm{O}\right)$, and ii $\left(2 \mathrm{H}_{2} \mathrm{O}\right)$ where the largest changes occur in the reactants and in the transition state zone; (b) reaction iii (both, $\mathrm{H}^{+}$and $\mathrm{H}_{3} \mathrm{O}^{+}$) where the changes happen in the reactants zone and the products zone; (c) reaction iv where the changes are in the transition state zone; and (d) reaction $\mathbf{v}$ where only the changes are in the reactants zone.

The $\mu$ profile of the reactions has been analyzed based on the charge of the systems considered. In the case of the $\mu$ profile of the reaction of the isolated neutral compounds and in the presence of water molecules (mechanisms i and ii) an almost linear increasing tendency is observed in the reactant region, while a deep reduction of $\mu$ is obtained in the TS region (Figure 4). A similar profile has been described in the literature by FloresMorales et al. $^{74}$ as the first step of the Maillard reaction. The profile of $\mu$ for mechanism $\mathbf{v}$ differs only in the small activity localized in the TS region. In all the neutral cases, the profiles are characterized by the presence of one maximum around or in the TS region (near $\xi_{1}$ for the $\mathbf{i}$ and ii and near $\xi_{\mathrm{TS}}$ for $\mathbf{v}$ ).

The profile of reaction in the cationic systems iii (both, $\mathrm{H}^{+}$and $\mathrm{H}_{3} \mathrm{O}^{+}$) show three critical points for the electronic chemical potential profile: two maxima, one before $\xi_{1}$ and another after $\xi_{2}$, and one minimum located around $\xi_{\mathrm{TS}}$. The TS area presents a low activity in this property. In addition, values of the $\mu$ are very different $\left(80-100 \mathrm{~kJ} \mathrm{~mol}^{-1}\right)$ for each of these reactions in contrast to what is observed in the neutral cases. Mechanism iv exhibits a completely different tendency with one minimum and one maximum in the limits where the TS area begins and ends.

The REF $[J(\xi)]$ analysis allows us to describe the changes in the slope of the electronic chemical potential (Figure 4). The mechanisms studied here present values near zero in the regions closed to $\xi_{\mathrm{R}}$ and $\xi_{\mathrm{P}}$. The only exception corresponds to the mechanism iii $\left(\mathrm{H}^{+}\right)$where the reaction starts and ends in a nonzero-flux regime. This indicates the instability of reactants and products and can be due to the fact that this is the only case where the reaction is not spontaneous.

The main changes in REF are located in the transition states and three kinds of behaviors can be found: (a) for $\mathbf{i}$, ii $\left(\mathrm{H}_{2} \mathrm{O}\right)$, and ii $\left(2 \mathrm{H}_{2} \mathrm{O}\right)$, REF has got a maximum around $\xi_{\mathrm{TS}}$ and with high values directly associated with the proton transfer occurred in this step; (b) for iii (both, $\mathrm{H}^{+}$and $\mathrm{H}_{3} \mathrm{O}^{+}$), REF decreases strongly from $\xi_{1}$ to $\xi_{2}$ by the formation of the $\mathrm{CO}$ covalent bond, which shows an enhanced electrophilicity; and (c) for iv and $\mathbf{v}$, REF increases strongly from $\xi_{1}$ to $\xi_{2}$.

All the mechanisms studied, except iv, start with a process of weakening of the $\mathrm{CO}$ bond, which is indicated by a negative flux in the reactants region. The change to a positive flux value is governed by processes of strengthening or bond formation: proton transfer with the formation of $\mathrm{OH}$ bond and $\mathrm{CO}$ bond formation are responsible for this stage. After that, reactions i, ii, and $\mathbf{v}$ do not experiment electronic activity. Mechanism iii exhibits two stationary points in REF: one maximum around $\xi_{1}$ that decays rapidly to one minimum around $\xi_{2}$. This fact indicates a poor synchronization in one process in which two stages are clearly separated: the formation of the $\mathrm{CO}$ bond followed by the proton transfer. Additionally, the REF values for reaction iii show negative values at the beginning of the products region, evolving latter on, toward positive values without reaching the nonzero flux regime for the iii $\left(\mathrm{H}^{+}\right)$reaction as have been discussed above.

In iv, the reaction starts with a positive flux, we attribute this to the increasing spontaneous reorganization of the electron density. Here, is also observed two critical points within the transition state region. However, in this case, first a minimum is obtained around $\xi_{1}$, followed by a region of almost constant values of REF, and a sudden maximum closed to $\xi_{2}$ is observed. In this mechanism, it is found, as previously, a poor synchronization of the two stages involved, and additionally, a reversed order of the breaking-forming bonds to what is observed in the acid catalyst mechanisms.

Local Index of Electrophilicity of the Carbon Atom of the Carbonyl Group. The local electrophilicity index of the carbon atom in formaldehyde (Figure 5) shows that there are four kinds of profiles. On the one hand, the mechanisms between the neutral systems show a high electrophilicity index value in the initial steps of the reactions. In mechanisms $\mathrm{i}$ and ii (both $\mathrm{H}_{2} \mathrm{O}$ and $2 \mathrm{H}_{2} \mathrm{O}$ ), the values at $\xi_{\mathrm{R}}$ are $12.5,10.8$, and $14.1 \mathrm{~kJ} \mathrm{~mol}^{-1}$, respectively. As the process progresses along the reaction coordinate, the values decrease to around $1.0-2.0 \mathrm{~kJ} \mathrm{~mol}^{-1}$ in the product zone, to the incipient formation of the new $\mathrm{CO}$ bond and the change of hybridization of the carbon atom from $\mathrm{sp}^{2}$ to $\mathrm{sp}^{3}$. In the case of mechanism $\mathbf{v}$, the electrophilicity index at $\xi_{\mathrm{R}}$ is $23.5 \mathrm{~kJ} \mathrm{~mol}^{-1}$, about twice the amount of that in the previous cases, i.e., in the synchronous process where methanol acts as nucleophilic agent with a molecule of formaldehyde as a proton donor to the other one, the electrophilicity index is enhanced in the initial stages of the reaction. It is important to note that, in the TS region, there is a change in the trend that, once the reaction enters in the region of the products, is again reversed. Also, the differences in the electrophilicity index between $\xi_{2}$ and $\xi_{1}$ $\left(\Delta \omega_{\mathrm{C}, \mathrm{TS}}\right)$ decrease with the size of the cycle, being $1.2 \mathrm{~kJ}$ $\mathrm{mol}^{-1}$ for i (four-membered ring), $0.8 \mathrm{~kJ} \mathrm{~mol}^{-1}$ for ii $\left(\mathrm{H}_{2} \mathrm{O}\right.$, sixmembered ring), and $0.5 \mathrm{~kJ} \mathrm{~mol}^{-1}$ for ii $\left(2 \mathrm{H}_{2} \mathrm{O}\right.$, eight-membered ring). This behavior is characteristic in all these neutral mechanisms, with the exception of $\mathbf{v}$ where the value of $\Delta \omega_{\mathrm{C}, \mathrm{TS}}$ is $-0.6 \mathrm{~kJ} \mathrm{~mol}^{-1}$. However, mechanisms iii $\left(\mathrm{H}^{+}\right)$, iii $\left(\mathrm{H}_{3} \mathrm{O}^{+}\right)$, and iv present electrophilicity profiles that are drastically different.

Mechanism iii $\left(\mathrm{H}^{+}\right)$starts with a higher value of electrophilicity index than in the neutral cases, $34.2 \mathrm{~kJ} \mathrm{~mol}^{-1}$ due to the effect of the $\mathrm{H}^{+}$cation clustered with the oxygen atom of the formaldehyde. However, from the TS region and up to $\xi_{\mathrm{p}}$, this property experiences an exponential growth character and, at this point, is $213.8 \mathrm{~kJ} \mathrm{~mol}^{-1}$. This huge value is expected because, as we explained when analyzing the reaction force and REF, as well as the geometry and the topology, a carbocation is formed. It is evident that the carbon atom in this species is one of the most powerful electrophilic chemical centers. In reaction iii $\left(\mathrm{H}_{3} \mathrm{O}^{+}\right)$, we anew find that the electrophilicity index is enhanced, and at $\xi_{\mathrm{R}}$, the value is $47.0 \mathrm{~kJ} \mathrm{~mol}^{-1}$. Ignoring the small fluctuation that occurs in the TS region (with a negative slope, i.e., in the opposite direction to that observed with neutral mechanisms $i$ and ii), we focus our attention on the region of the products. In this case, $\Delta \omega_{\mathrm{C}}\left[\xi_{1}\right]>\Delta \omega_{\mathrm{C}}\left[\xi_{2}\right]$, with a value of $29.2 \mathrm{~kJ} \mathrm{~mol}^{-1}$ for the last one. We can affirm again that, although the new $\mathrm{sp}^{3}$ carbon remains activated, the water molecule is not eliminated. Finally, the profile for iv is quite interesting for two reasons: the first one 
because its evolution shows a completely different profile, and the second one because anomalous values of electrophilicity index appear. Concerning the profile, it increases from $\xi_{\mathrm{R}}$ to $\xi_{1}$, and immediately decreases from $\xi_{1}$ to $\xi_{\mathrm{TS}}$, a point at which it increases again to slightly enter the products region where once more it decreases. The range of electrophilicity index is situated between 1.0 and $6.0 \mathrm{~kJ} \mathrm{~mol}^{-1}$, the lowest in all the reactions. This fact is the response to the shortest $\mathrm{C} \cdot \cdots \mathrm{O}$ distance characterized since the beginning of the reaction, in other words, the bridge water molecule as well as the cation $\mathrm{H}_{3} \mathrm{O}^{+}$have transformed the reactants in a stable reactive complex where the carbon atom has already lost too much its $\mathrm{sp}^{2}$ character.

\section{CONCLUSIONS}

A theoretical study of the hemiacetal formation between formaldehyde and methanol has been carried out. The reaction in the gas phase between the isolated monomers shows a high TS barrier $\left(141.3 \mathrm{~kJ} \mathrm{~mol}^{-1}\right)$. Two types of catalysis have been considered: inclusion of an acid moiety $\left(\mathrm{H}^{+}\right.$or $\left.\mathrm{H}_{3} \mathrm{O}^{+}\right)$interacting with the carbonyl moiety and the use of assistants, especially bridging water molecules, in the proton transfer process. The lowest barriers are obtained in the first case with the $\mathrm{H}_{3} \mathrm{O}^{+}$system $\left(86.5 \mathrm{~kJ} \mathrm{~mol}^{-1}\right)$ and with a single water molecule $\left(74.7 \mathrm{~kJ} \mathrm{~mol}^{-1}\right)$ in the second. The inclusion of both effects simultaneously provides a very small barrier $\left(25.0 \mathrm{~kJ} \mathrm{~mol}^{-1}\right)$. In the autoassisted mechanism, where two pairs of reactants interact forming a $C_{i}$ symmetry tetramer and the proton transfer is assisted by the second pair of reactants, we found the smallest value of the barrier in the neutral reaction considered here, $61.2 \mathrm{~kJ} \mathrm{~mol}^{-1}$.

The analysis of the electronic contribution to the TS shows that, in the neutral systems, it corresponds between 7 and $12 \%$ of the total value, while for the reaction catalyzed by acids, it increases to values between 28 and $36 \%$.

The changes in REF are located in the transition states, and three kinds of behaviors can be found: (a) for i, ii $\left(\mathrm{H}_{2} \mathrm{O}\right)$, and ii $\left(2 \mathrm{H}_{2} \mathrm{O}\right)$, REF has a maximum around $\xi_{\mathrm{TS}}$ and with high values directly associated with the proton transfer occurred in this step; (b) for iii (both, $\mathrm{H}^{+}$and $\mathrm{H}_{3} \mathrm{O}^{+}$), REF decreases strongly from $\xi_{1}$ to $\xi_{2}$ by the formation of the CO covalent bond, which shows an enhanced electrophilicity; and (c) for iv and $\mathbf{v}$, REF increases strongly from $\xi_{1}$ to $\xi_{2}$.

The presence of two stationary points of the REF profile in the transition state regions of the cationic (acid) mechanisms lead us to conclude that the two principal stages involved in this hemiacetal formation show a poor synchronicity, contrary to what was observed in the neutral mechanisms.

\section{ASSOCIATED CONTENT}

\section{S Supporting Information}

Geometries of the stationary points (energy and reaction force) of the reactions $\mathbf{i}$, ii (both $\mathrm{H}_{2} \mathrm{O}$ and $2 \mathrm{H}_{2} \mathrm{O}$ ), and $\mathbf{v}$; geometries of the TSs; energy and reaction force vs. reaction coordinate calculated at the B3LYP/6-311++G(d,p) computational level. This material is available free of charge via the Internet at http:// pubs.acs.org.

\section{AUTHOR INFORMATION}

\section{Corresponding Author}

*E-mail: ibon@iqm.csic.es. Webpage: http://www.iqm.csic.es/ are.

\section{Notes}

The authors declare no competing financial interest.

\section{ACKNOWLEDGMENTS}

L.M.A. thanks the Ministerio de Ciencia e Innovación for a Ph.D. grant (No. BES-2010-031225). We also thank the Ministerio de Ciencia e Innovación (Project No. CTQ2009-13129-C02-02) and the Comunidad Autónoma de Madrid (Project MADRISOLAR2, ref S2009/PPQ-1533) for continuing support. A.T.L. wishes to thank FONDECYT through Project No. 1090460 for financial support. Thanks are also given to the CTI (CSIC) for an allocation of computer time.

\section{REFERENCES}

(1) Michael, J. M.; Smith, B. Advanced Organic Chemistry: Reactions, Mechanisms and Structure, 5th ed.; John Wiley \& Sons: New York, 2001.

(2) Stewart, R. The Proton: Applications to Organic Chemistry; Academic Press: Orlando, FL, 1985.

(3) Sorensen, P. E.; Jencks, W. P. J. Am. Chem. Soc. 1987, 109, 46754690.

(4) Funderburk, L. H.; Aldwin, L.; Jencks, W. P. J. Am. Chem. Soc. 1978, 100, 5444-5459.

(5) Meijer, E. J.; Sprik, M. J. Am. Chem. Soc. 1998, 120, 6345-6355.

(6) Minyaev, R. Russ. Chem. Bull. 1998, 47, 8-16.

(7) Conner, A. H. J. Appl. Polym. Sci. 2000, 78, 355-363.

(8) Schmidt, R. K.; Karplus, M.; Brady, J. W. J. Am. Chem. Soc. 1996, 118, 541-546.

(9) Yamabe, S.; Ishikawa, T. J. Org. Chem. 1999, 64, 4519-4524.

(10) Morpurgo, S.; Brahimi, M.; Bossa, M.; Morpurgo, G. O. Phys. Chem. Chem. Phys. 2000, 2, 2707-2713.

(11) Morpurgo, S.; Bossa, M. Phys. Chem. Chem. Phys. 2003, 5, 11811189.

(12) Morpurgo, S.; Grandi, A.; Zazza, C.; Bossa, M. THEOCHEM 2005, 729, 71-82.

(13) Silva, A. M.; da Silva, E. C.; da Silva, C. O. Carbohydr. Res. 2006, 341, 1029-1040.

(14) Lewis, B. E.; Choytun, N.; Schramm, V. L.; Bennet, A. J. J. Am. Chem. Soc. 2006, 128, 5049-5058.

(15) Silva, C. Theor. Chem. Acc. 2006, 116, 137-147.

(16) Alkorta, I.; Popelier, P. L. A. Carbohydr. Res. 2011, 346, $2933-$ 2939.

(17) Fragoso-Serrano, M.; Pereda-Miranda, R.; Cerda-Garcia-Rojas, C. M. Tetrahedron 2006, 62, 11916-11924.

(18) Caldin, E. F.; Gold, V. Proton Transfer Reactions; Chapman and Hall: London, U.K., 1975.

(19) Zundel, G. In Advances in Chemical Physics; John Wiley \& Sons, Inc.: New York, 2007; pp 1-217.

(20) Ruiz-Pernía, J. J.; Alves, C. N.; Moliner, V.; Silla, E.; Tuñón, I. THEOCHEM 2009, 898, 115-120.

(21) Ardèvol, A.; Rovira, C. Angew. Chem., Int. Ed. 2011, 50, 1089710901.

(22) Eigen, M. Angew. Chem., Int. Ed. 1964, 3, 1-19.

(23) Lin, Y.; Wang, H.; Gao, S.; Schaefer, H. F. J. Phys. Chem. B 2011, $115,11746-11756$

(24) Chen, P.-T.; Wang, C.-C.; Jiang, J.-C.; Wang, H.-K.; Hayashi, M. J. Phys. Chem. B 2011, 115, 1485-1490.

(25) Vilciauskas, L.; Paddison, S. J.; Kreuer, K.-D. J. Phys. Chem. A 2009, 113, 9193-9201.

(26) Yu, R; De Jonghe, L. C. J. Phys. Chem. C 2007, 111, 1100311007.

(27) Sengupta, P. K.; Kasha, M. Chem. Phys. Lett. 1979, 68, 382-385. (28) Barbatti, M.; Aquino, A. J. A.; Lischka, H.; Schriever, C.; Lochbrunner, S.; Riedle, E. Phys. Chem. Chem. Phys. 2009, 11, 14061415.

(29) Leiderman, P.; Genosar, L.; Huppert, D.; Shu, X.; Remington, S. J.; Solntsev, K. M.; Tolbert, L. M. Biochemistry 2007, 46, 12026-12036.

(30) Tolbert, L. M.; Solntsev, K. M. Acc. Chem. Res. 2001, 35, 19-27.

(31) Douhal, A.; Lahmani, F.; Zewail, A. H. Chem. Phys. 1996, 207, 477-498. 
(32) Carlier, P.; Hannachi, H.; Mouvier, G. Atmos. Environ. 1986, 20, 2079-2099.

(33) Bruna, P. J.; Hachey, M. R. J.; Grein, F. THEOCHEM 1997, 400, 177-221.

(34) Raúl Alvarez-Idaboy, J.; Mora-Diez, N.; Boyd, R. J.; Vivier-Bunge, A. J. Am. Chem. Soc. 2001, 123, 2018-2024.

(35) Jiao, Z.; Luo, P.; Wu, Y.; Ding, S.; Zhang, Z. J. Hazard. Mater. 2006, 134, 176-182.

(36) Rincón, E.; Toro-Labbé, A. Chem. Phys. Lett. 2007, 438, 93-98.

(37) Politzer, P.; Toro-Labbé, A.; Gutiérrez-Oliva, S.; Herrera, B.; Jaque, P.; Concha, M.; Murray, J. J. Chem. Sci. 2005, 117, 467-472.

(38) Gutiérrez-Oliva, S.; Herrera, B.; Toro-Labbé, A.; Chermette, H. J. Phys. Chem. A 2005, 109, 1748-1751.

(39) Toro-Labbe, A.; Gutierrez-Oliva, S.; Concha, M. C.; Murray, J. S.; Politzer, P. J. Chem. Phys. 2004, 121, 4570-4576.

(40) Martínez, J.; Toro-Labbé, A. Chem. Phys. Lett. 2004, 392, 132139.

(41) Jaque, P.; Toro-Labbé, A. J. Phys. Chem. A 2000, 104, 995-1003.

(42) Toro-Labbé, A. J. Phys. Chem. A 1999, 103, 4398-4403.

(43) Herrera, B.; Toro-Labbé, A. J. Phys. Chem. A 2007, 111, 59215926.

(44) Echegaray, E.; Toro-Labbé, A. J. Phys. Chem. A 2008, 112, 1180111807.

(45) Vogt-Geisse, S.; Toro-Labbé, A. J. Chem. Phys. 2009, 130, 244308.

(46) Parr, R. G.; Szentpály, L. V.; Liu, S. J. Am. Chem. Soc. 1999, 121,

1922-1924.

(47) Fukui, K. J. Phys. Chem. 1970, 74, 4161-4163.

(48) Fukui, K. Acc. Chem. Res. 1981, 14, 363-368.

(49) Laidler, K. J.; King, M. C. J. Phys. Chem. 1983, 87, 2657-2664.

(50) Parr, R. G.; Yang, W. Density-Functional Theory of Atoms and Molecules; Oxford University Press: Oxford, U.K., 1994.

(51) Geerlings, P.; De Proft, F.; Langenaeker, W. Chem. Rev. 2003, 103, $1793-1874$.

(52) Koopmans, T. Physica 1934, 1, 104.

(53) Parr, R. G.; Donnelly, R. A.; Levy, M.; Palke, W. E. J. Chem. Phys. 1978, 68, 3801-3807.

(54) Parr, R. G.; Pearson, R. G. J. Am. Chem. Soc. 1983, 105, 75127516.

(55) Pearson, R. J. Chem. Sci. 2005, 117, 369-377.

(56) Hohenberg, P.; Kohn, W. Phys. Rev. 1964, 136, B864-B871.

(57) Toro-Labbé, A. Theoretical Aspects of Chemical Reactivity; Elsevier: Amsterdam, The Netherlands, 2007; Vol. 19.

(58) Cerón, M. L.; Echegaray, E.; Gutiérrez-Oliva, S.; Herrera, B.; Toro-Labbé, A. Sci. Chin. Chem. 2011, 54, 1982-1988.

(59) Becke, A. D. J. Chem. Phys. 1993, 98, 5648-5652.

(60) Lee, C. T.; Yang, W. T.; Parr, R. G. Phys. Rev. B 1988, 37, 785789.

(61) Frisch, M. J.; Pople, J. A.; Binkley, J. S. J. Chem. Phys. 1984, 80, 3265-3269.

(62) Peng, C.; Ayala, P. Y.; Schlegel, H. B.; Frisch, M. J. J. Comput. Chem. 1996, 17, 49-56.

(63) Gonzalez, C.; Schlegel, H. B. J. Phys. Chem. 1990, 94, 5523-5527.

(64) Frisch, M. J.; Trucks, G. W.; Schlegel, H. B.; Scuseria, G. E.; Robb, M. A.; Cheeseman, J. R.; Scalmani, G.; Barone, V.; Mennucci, B.; Petersson, G. A.; Nakatsuji, H.; Caricato, M.; Li, X.; Hratchian, H. P.; Izmaylov, A. F.; Bloino, J.; Zheng, G.; Sonnenberg, J. L.; Hada, M.; Ehara, M.; Toyota, K.; Fukuda, R.; Hasegawa, J.; Ishida, M.; Nakajima, T.; Honda, Y.; Kitao, O.; Nakai, H.; Vreven, T.; Montgomery, J. A., Jr.; Peralta, J. E.; Ogliaro, F.; Bearpark, M.; Heyd, J. J.; Brothers, E.; Kudin, K. N.; Staroverov, V. N.; Kobayashi, R.; Normand, J.; Raghavachari, K.; Rendell, A.; Burant, J. C.; Iyengar, S. S.; Tomasi, J.; Cossi, M.; Rega, N.; Millam, J. M.; Klene, M.; Knox, J. E.; Cross, J. B.; Bakken, V.; Adamo, C.; Jaramillo, J.; Gomperts, R.; Stratmann, R. E.; Yazyev, O.; Austin, A. J.; Cammi, R.; Pomelli, C.; Ochterski, J. W.; Martin, R. L.; Morokuma, K.; Zakrzewski, V. G.; Voth, G. A.; Salvador, P.; Dannenberg, J. J.; Dapprich, S.; Daniels, A. D.; Farkas, O.; Foresman, J. B.; Ortiz, J. V.; Cioslowski, J.; Fox, D. J. Gaussian 09, revision A.02; Gaussian, Inc.: Wallingford, CT, 2009.
(65) Weinhold, F.; Landis, C. R. Valency and Bonding. A Natural Bond Orbital Donor-Aceptor Perspective; Cambridge University Press: Cambridge, U.K., 2005.

(66) Glendening, E. D.; Reed, A. E.; Carpenter, J. E.; Weinhold, F. NBO, version 3.1; Gaussian, Inc.: Wallingford, CT, 2009.

(67) Bader, R. F. W. Atoms in Molecules: A Quantum Theory; Clarendon Press: Oxford, U.K., 1990.

(68) Popelier, P. L. A. Atoms In Molecules. An Introduction; Prentice Hall: Harlow, U.K., 2000.

(69) Popelier, P. L. A.; Bone, R. G. A. MORPHY98, A Topological Analysis Program, 1999.

(70) Popelier, P. L. A. Chem. Phys. Lett. 1994, 228, 160-164.

(71) Casadei, M. A.; Galli, C.; Mandolini, L. J. Am. Chem. Soc. 1984, $106,1051-1056$.

(72) Abboud, J.-L. M.; Alkorta, I.; Dávalos, J. Z.; Müller, P.; Quintanilla, E. Advances in Physical Organic Chemistry; Academic Press: New York, 2002; Vol. 37, pp 57-135.

(73) Esseffar, M.; El Mouhtadi, M.; López, V.; Yáñez, M. THEOCHEM 1992, 255, 393-408.

(74) Flores-Morales, P.; Gutiérrez-Oliva, S.; Silva, E.; Toro-Labbé, A. THEOCHEM 2010, 943, 121-126. 